\title{
Is jugular bulb stenosis in vein of Galen aneurysmal malformation associated with bony remodeling of the jugular foramina?
}

\author{
Guillaume Saliou, MD, PhD, ${ }^{1,2}$ Peter Dirks, MD, PhD, ${ }^{3}$ Lee-Anne Slater, MBBS, MMed, ${ }^{1}$ and \\ Timo Krings, MD, PhD, FRCP(C) ${ }^{1}$
} 1Neuroradiology, Toronto Western Hospital \& University Health Network; and ${ }^{3}$ Division of Neurosurgery, The Hospital for Sick
Children, Toronto, Ontario, Canada; and ${ }^{2}$ Neuroradiologie, CHU Bicêtre, Le Kremlin Bicêtre, France

\begin{abstract}
OBJECTIVE The etiology of jugular bulb stenosis (JBS) or occlusion in the context of vein of Galen aneurysmal malformations (VGAMs) is unknown. It can lead to decompensation of a lesion that was previously clinically stable. The aim of this study was to describe the natural history of JBS or occlusion in VGAM and to determine whether there is an association with bony remodeling of the jugular foramina.
\end{abstract}

METHODS The authors identified all cases of JBS greater than $70 \%$ bilaterally involving patients seen at The Hospital for Sick Children between January 2007 and June 2014. The foramen diameters were measured on sagittal CT imaging, on a slice passing at the level of the jugular vein. The jugular foramen diameters were also compared to measurements obtained in a matched population of the same age group who had no VGAM and had undergone cerebral CT for a reason other than vascular disease.

RESULTS Eight patients ( 6 male and 2 female) with bilateral JBS were included in this series. The median duration of clinical follow-up was 2.5 years (IQR 1.7-4.2 years). JBS was associated with bony narrowing the jugular foramina in 7 of the 8 patients over time. Between 1 and 2 years of age, patients with a VGAM demonstrated jugular foramen narrowing in comparison with a matched population $(p=0.015)$.

CONCLUSIONS Jugular bulb stenosis or occlusion in VGAM may be associated with narrowing of the jugular foramina. These conditions seem to have a male predominance. If treatment is required, bony narrowing of the jugular foramina should be taken into account when deciding whether angioplasty and stent placement or surgical bypass might be appropriate therapeutic options.

http://thejns.org/doi/abs/10.3171/2015.12.PEDS15310

KEY WORDS vein of Galen aneurysmal malformation; jugular bulb stenosis; angioarchitecture; vascular disorders

$\mathrm{V}$ EIN of Galen aneurysmal malformations (VGAMs) are rare congenital arteriovenous malformations of the choroidal system. ${ }^{1}$ In true VGAMs, arteriovenous fistulas drain into the aberrantly persistent embryonic precursor of the vein of Galen, the fetal median prosencephalic vein of Markowski. ${ }^{12}$ The deep venous system drains through persistent alternative embryonic routes, mainly the lateral mesencephalic and lateral pontine veins and the superior petrosal sinus. ${ }^{11}$ Although the natural history of VGAM is unclear, these lesions can cause severe morbidity and high mortality rates. . $, 4,5,7,8^{-}$
While jugular bulb narrowing is exceedingly rare at birth, as demonstrated in the literature ${ }^{14}$ it can be seen on follow-up imaging in a significant number of patients with VGAM.13,14 The etiology of jugular bulb stenosis (JBS) or occlusion in the context of VGAM is, however, unknown. When it occurs, it can lead to decompensation of a previously clinically stable lesion due to intracranial venous congestion, chronic cerebral venous ischemia, hydrovenous disorders, or cerebral hemorrhage. ${ }^{9,10}$ JBS also leads to an increased risk of extensive venous thrombosis in the days following transarterial embolization adding

ABBREVIATIONS BOS = Bicêtre Outcome Score; IQR = interquartile range; JBS = jugular bulb stenosis; VGAM = vein of Galen aneurysmal malformation. SUBMITTED May 19, 2015. ACCEPTED December 7, 2015.

INCLUDE WHEN CITING Published online April 1, 2016; DOI: 10.3171/2015.12.PEDS15310. 
complexity to the treatment of VGAMs.$^{13}$ Our aim was to determine whether there is an association with bony remodeling of the jugular foramina.

\section{Methods}

\section{Patient Population}

Data were extracted from a single-center database of The Hospital for Sick Children. Thirty-five consecutive cases involving children presenting with a true VGAM between January 2007 and June 2014 were identified. From this cohort, those patients with severe jugular stenosis were studied, as in our department all patients with a VGAM who present with severe JBS undergo a CT scan to assess the size of the jugular foramina in case endovascular treatment of the stenosis was required. If no significant JBS was noted in the follow-up imaging studies (MRI or digital subtraction angiography), no CT scan was indicated and noninvasive imaging follow-up was performed with MRI and MR angiography (MRA) only. Thus there was no CT assessment of the size of the jugular foramina in the population without severe JBS, so bony foraminal changes could not be analyzed in those patients.

\section{Clinical Features}

The following data were collated for each patient: sex, type of vein of Galen (i.e., choroidal or mural), and Bicêtre Outcome Score (BOS) ${ }^{9,10}$ assessed at the last clinical assessment.

\section{Radiological Features}

Conventional MRI and CT scans performed at birth when available, between 4 and 6 months, and between 12 and 24 months, were reviewed.

The percentage of JBS was assessed on MRA (2D time-of-flight [TOF] sequence) as the smallest diameter of the jugular bulb on a sagittal/lateral view over the diameter of the jugular vein $2 \mathrm{~cm}$ below the jugular foramina (Fig. 1). As there is no consensus regarding what per- centage stenosis equates to clinical significance, we used a previously published threshold value of $70 \%{ }^{6}$ We only determined a stenosis of greater than $70 \%$ to be significant if there was involvement of the bilateral jugular bulbs. The values for the measurements of the jugular bulbs are presented as the mean of the stenosis on both sides, with the individual measurements for the right and left presented in parentheses.

The diameter of the jugular foramina was measured on sagittal CT imaging, on a slice passing at the level of the jugular vein. We also compared the jugular foramina diameters to measurements obtained in a matched population of 20 patients of the same age groups (i.e., 20 patients between 4 and 6 months old and 20 patients between 1 and 2 years old) who had no VGAM and had undergone cerebral CT for a reason other than vascular disease.

\section{Statistical Analysis}

Descriptive statistics were calculated to summarize the distribution of JBS or narrowing of the jugular foramina at 4-6 months and 1-2 years of age (Table 1). The distribution of categorical variables was described by frequencies and percentages; the distribution of continuous and non-normally distributed variables was described by medians and interquartile ranges (IQRs). Univariate statistics were performed to assess for associations between jugular foramen narrowing and VGAMs at 4-6 months and 1-2 years of age. These were performed via Wilcoxon-MannWhitney test. A p value $<0.05$ was considered statistically significant.

\section{Ethical Issues}

This medical chart and imaging review was conducted after approval by our local research and ethics board. This was a retrospective noninterventional study. No blood tests or clinical investigations were required beyond those required for the standard care of these patients. The study was performed in accordance with our ethical guidelines.
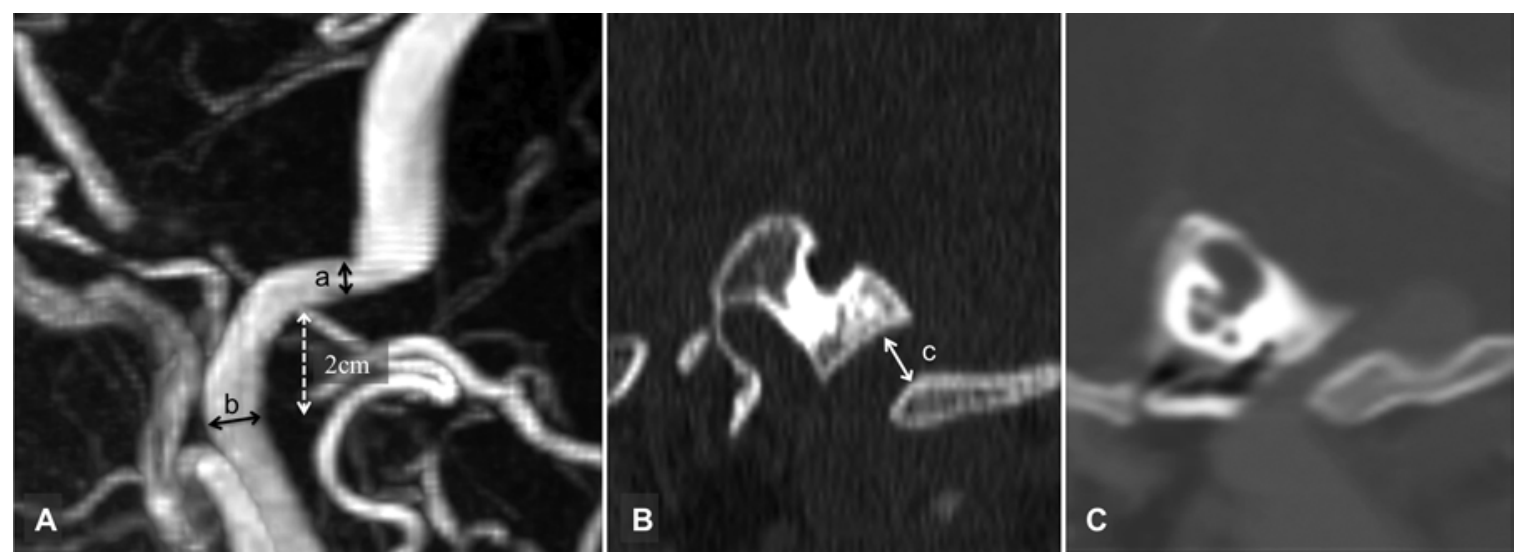

FIG. 1. A: Venous MR angiogram showing measurement of the stenosis at the jugular bulb level. The area of maximum stenosis is indicated by $a$ and the diameter of the jugular vein $2 \mathrm{~cm}$ below the jugular foramen, by $b$. B: CT scan showing measurement of the diameter of the jugular foramen in a patient without VGAM (matched population). The sagittal diameter of the jugular foramina at the level of the jugular vein is indicated by $c$. C: CT scan showing an example of jugular foramen narrowing among our population (Case 7; scan obtained when the patient was between 1 and 2 years old). 


\section{Results}

Of the 35 children, 8 had bilateral jugular bulb narrowing, and these patients were included in this series. The median duration of clinical follow-up was 2.5 years (IQR 1.7-4.2 years). Six (75\%) of the patients were male and 2 $(25 \%)$ were female.

The patients' median age at initial presentation was 3.6 months (IQR 1.7-4.8 months). Only 2 patients (Cases 1 and 5) had imaging studies performed in the 1st month of life (Day 1 for both), and no JBS was observed in those studies. The patients' median age at 4-6 months imaging was 4.8 months (IQR 4.4-5.3 months). Only 4 patients had bilateral significant JBS on these imaging studies (median percentage of stenosis: 92\% [IQR 89.5\%-94.5\%]). The 4 remaining patients had no stenosis (3 patients) or mild stenosis (1 patient had $85 \%$ stenosis on the right side and $50 \%$ on the left side). In the cases of bilateral JBS, the stenosis was identified when the patients were between 1 and 2 years of life (median 14.7 months [IQR 13.4-16 months]) with a median stenosis of 96\% (IQR 77\%-100\%).

JBS was associated with bony narrowing of the jugular foramina in 7 of the 8 patients over time. In these 7 patients, the median diameter of the jugular foramina was $3.4 \mathrm{~mm}$ (IQR 2.4-4 mm) at 4-6 months of age and $3 \mathrm{~mm}$ (IQR 2.4-3.9 mm) at 1-2 years of age (Fig. 1). In the patient who did not demonstrate narrowing of the jugular foramina, the diameters of the foramina were $3.6 \mathrm{~mm} \mathrm{bi-}$ laterally at 6 months of age and $7 \mathrm{~mm}$ at 1.2 years of age despite no improvement in JBS.

In the matched controls, the mean diameter of jugular foramina was $3.9 \mathrm{~mm}$ (IQR 3.3-4.2 mm) at 4-6 months and $5.1 \mathrm{~mm}$ (IQR 4.4-5.6 mm) at 1-2 years. At 4-6 months there was no significant difference between the mean diameter in our population and the matched population. However, in the studies obtained when the patients were 1-2 years of age, the jugular foramina were significantly narrower in patients with a VGAM than in the matched controls $(\mathrm{p}=0.015)$.

The patients' median age at the last clinical follow-up was 2.5 years (IQR 1.7-4.2 years), and the BOS was 1 in 1 patient, 2 in 2 patients, 4 in 3 patients, and 5 in 2 patients.

The main findings are summarized in Tables 1-3.

\section{Discussion}

The cause of the jugular bulb stenosis or occlusion is unknown. The influence of abnormal skull base growth maturation caused by macrocrania as opposed to a venous high-flow angiopathy has been hypothesized. ${ }^{9,14}$ In the present series, we were not able to assess whether the jugular bulb was narrowing prior to the jugular foramen. However, we observed 1 patient (Case 2) without bony narrowing and 2 patients (Cases 1 and 5) with no or limited bony narrowing on one side despite severe JBS. Thus, we hypothesize based on these limited observations that venous high-flow angiopathy seems to precede the bony foraminal narrowing. The bony narrowing may thus be a secondary phenomenon following the decreased jugular bulb diameter but may be absent in rare cases (only 1 patient in the present series).

We did not find any angiomorphological features that

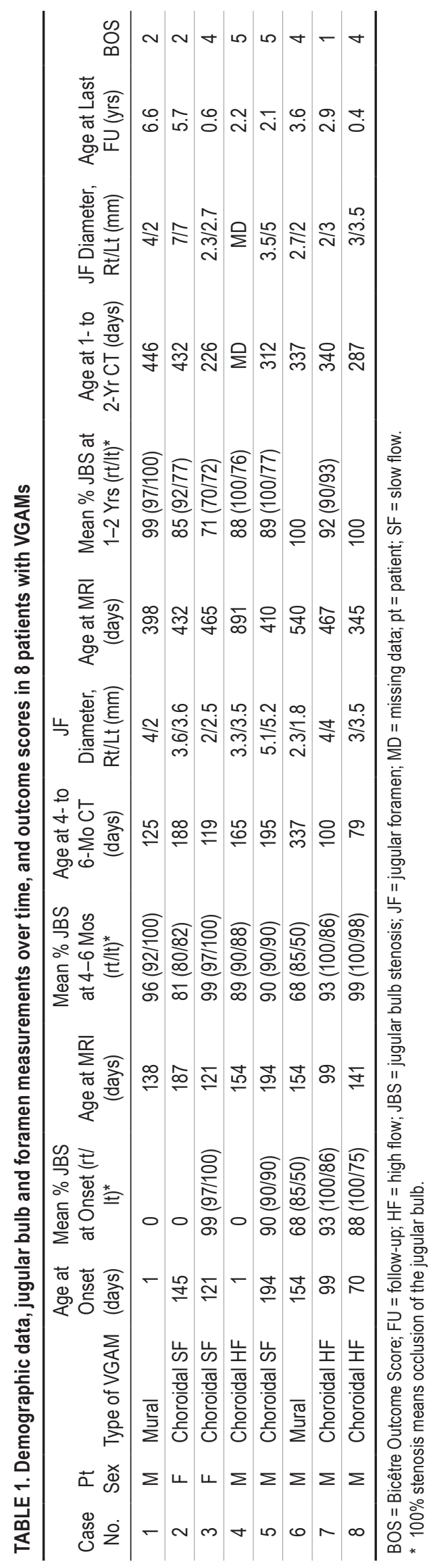


TABLE 2. Demographic data and results of tests of interest in patients in the present series and the matched population

\begin{tabular}{|c|c|c|c|}
\hline Variable & Present Series & Comparison Population & $\mathrm{p}$ Value \\
\hline No. of pts & 8 & 20 & \\
\hline \multicolumn{4}{|l|}{ Data at 4-6 mos } \\
\hline Median age at imaging (mos) & 4.8 (IQR 4.4-5.3) & 5 (IQR 4-5.3) & NS \\
\hline Mean diameter of jugular foramina (mm) & 3 (IQR 2.4-4) & 3.9 (IQR 3.3-4.2) & NS \\
\hline \multicolumn{4}{|l|}{ Data at $1-2$ yrs } \\
\hline Median age at imaging (mos) & 14.7 (IQR 13.4-16) & 16 (IQR 14-18) & NS \\
\hline Mean diameter of jugular foramina (mm) & 3 (IQR 2.4-3.9) & 5.1 (IQR 4.4-5.6) & 0.015 \\
\hline
\end{tabular}

NS = not significant.

could be used to predict jugular foramen stenosis when comparing the current group to the remainder of VGAM patients who did not present with JBS.

If the JBS is severe, the VGAM and the brain may drain into the same cavernous sinus outlets. Thus some degree of cerebral pial congestion may exist without reflux. ${ }^{9}$ The long-term result is a chronic ischemic phenomenon with delayed calcifications and the peculiar "pseudo-phlebitic" appearance of the cortical veins. This condition can lead to a very poor outcome, and may even appear if the vein of Galen arteriovenous shunt is completely cured. This is illustrated in the case reported by Brew et al., in which a patient with a VGAM that was successfully treated with complete embolization of the shunt died 1 year after treatment following total occlusion of the jugular bulbs in the absence of alternative venous outflow pathways. ${ }^{3}$ In our experience, we have also had a patient with a VGAM in Bicêtre (not reported) with bilateral jugular bulb occlusion who, despite complete occlusion of the VGAM shunts, experienced delayed hydrovenous complications, including hydrocephalus requiring shunting as well as cerebral venous thrombosis. This patient subsequently died of these complications 2 years following jugular bulb occlusion.

Our series demonstrated that JBS is associated with narrowing of the jugular foramina in most cases. This may have implications for treatment. There is currently

\section{TABLE 3. Bicêtre Outcome Score}

\begin{tabular}{cl}
\hline Score & \multicolumn{1}{c}{ Description } \\
\hline 0 & Death \\
\hline 1 & $\begin{array}{c}\text { Severe neurological symptoms, mental retardation of more } \\
\text { than } 20 \%, \text { specialized school, and/or cardiac failure } \\
\text { unstable despite treatment }\end{array}$ \\
\hline 2 & $\begin{array}{c}\text { Permanent minor neurological symptoms, mental retardation } \\
\text { of up to 20\%, nonpermanent neurological symptoms under } \\
\text { treatment, normal school with support, and/or cardiac } \\
\text { failure stabilized with treatment }\end{array}$ \\
\hline 3 & $\begin{array}{c}\text { Transient neurological symptoms, not treated, and/or asymp- } \\
\text { tomatic cardiac overload under treatment }\end{array}$ \\
\hline 4 & $\begin{array}{c}\text { Minimal non-neurological symptoms, not treated, and/or } \\
\text { asymptomatic enlargement of the cardiac silhouette }\end{array}$ \\
\hline 5 & Normal \\
\hline
\end{tabular}

only 1 article reporting angioplasty of the jugular vein in VGAM,${ }^{3}$ but this treatment may be considered as a therapeutic option as JBS precedes the bony changes. Prior to any such treatment, we recommend evaluation for bony stenosis, as bony constriction may hinder successful dilation of the vessel. If bony changes are, however, only occurring secondarily after initial venous stenosis, angioplasty may be warranted to avoid secondary irreversible bony changes. In the presence of bony stenosis a bypass may be considered as an alternative therapy.

JBS was not seen at birth in our limited series, as previously reported. ${ }^{14}$ The 2 patients with early imaging (1st day of life) had no evidence of JBS. Stenoses seemingly develop later, during the first months of life. JBS was not only associated with high-flow fistulas, as in our cohort 3 patients presented with a slow-flow choroidal VGAM and 5 patients with high-flow shunts. Additionally, at 4-6 months of age, there was no predominance of stenosis in the patients with high-flow VGAMs compared with those with slow-flow lesions.

This study has the inherent limitations of a retrospective study. Moreover, given the small size of the population, we are unable to draw any firm conclusions regarding the nature and exact timing of JBS. However, we believe that this small observational study demonstrates some potentially clinically relevant and pertinent findings with therapeutic implications.

\section{Conclusions}

In case of JBS or occlusion in VGAM, a CT scan should be performed to identify whether there is any associated bony narrowing. JBS or occlusion in VGAM seems to have a male predominance. Mechanisms are still poorly understood, and our study did not identify any angiomorphological features of VGAMs that predicted occurrence of this complication. In cases in which treatment is required, bony narrowing of the jugular foramina should be taken into account when deciding whether angioplasty and stenting or surgical bypass might be appropriate therapeutic options.

\section{References}

1. Alvarez H, Garcia Monaco R, Rodesch G, Sachet M, Krings T, Lasjaunias P: Vein of Galen aneurysmal malformations. Neuroimaging Clin N Am 17:189-206, 2007 
2. Berenstein A, Masters LT, Nelson PK, Setton A, Verma R: Transumbilical catheterization of cerebral arteries. Neurosurgery 41:846-850, 1997

3. Brew S, Taylor W, Reddington A: Stenting of a venous stenosis in vein of Galen aneurysmal malformation. A case report. Interv Neuroradiol 7:237-240, 2001

4. Dahdah NS, Alesseh H, Dahms B, Saker F: Severe pulmonary hypertensive vascular disease in two newborns with aneurysmal vein of Galen. Pediatr Cardiol 22:538-541, 2001

5. Frawley GP, Dargaville PA, Mitchell PJ, Tress BM, Loughnan P: Clinical course and medical management of neonates with severe cardiac failure related to vein of Galen malformation. Arch Dis Child Fetal Neonatal Ed 87:F144-F149, 2002

6. Geibprasert S, Krings T, Armstrong D, terBrugge KG, Raybaud CA: Predicting factors for the follow-up outcome and management decisions in vein of Galen aneurysmal malformations. Childs Nerv Syst 26:35-46, 2010

7. Johnston IH, Whittle IR, Besser M, Morgan MK: Vein of Galen malformation: diagnosis and management. Neurosurgery 20:747-758, 1987

8. Jones BV, Ball WS, Tomsick TA, Millard J, Crone KR: Vein of Galen aneurysmal malformation: diagnosis and treatment of 13 children with extended clinical follow-up. AJNR Am J Neuroradiol 23:1717-1724, 2002

9. Lasjaunias P, Berenstein A, terBrugge KG: Vein of Galen aneurysmal malformation, in Surgical Neuroangiography 3. Clinical and Interventional Aspects in Children, ed 2. Berlin: Springer, 2006, pp 105-226

10. Lasjaunias PL, Chng SM, Sachet M, Alvarez H, Rodesch G, Garcia-Monaco R: The management of vein of Galen aneurysmal malformations. Neurosurgery 59 (Suppl 3):S184S194, S3-S13, 2006

11. Lasjaunias P, Garcia-Monaco R, Rodesch G, terBrugge K: Deep venous drainage in great cerebral vein (vein of Galen) absence and malformations. Neuroradiology 33:234-238, 1991

12. Markowski J: Entwicklung der Sinus durae matris und der Hirnvenen des Menschen. Bull Acad Sci Lett B:1-269, 1921

13. Puccinelli F, Deiva K, Bellesme C, Durand P, Chevret L, Husson B, et al: Cerebral venous thrombosis after embolization of pediatric AVM with jugular bulb stenosis or occlusion: management and prevention. Eur J Paediatr Neurol 18:766-773, 2014

14. Raybaud CA, Hald JK, Strother CM, Choux M, Jiddane M: [Aneurysms of the vein of Galen. Angiographic study and morphogenetic considerations.] Neurochirurgie 33:302-314, $1987(\mathrm{Fr})$

\section{Disclosures}

The authors report no conflict of interest concerning the materials or methods used in this study or the findings specified in this paper.

\section{Author Contributions}

Conception and design: Saliou, Dirks, Krings. Acquisition of data: all authors. Analysis and interpretation of data: all authors. Drafting the article: all authors. Critically revising the article: all authors. Reviewed submitted version of manuscript: all authors. Approved the final version of the manuscript on behalf of all authors: Saliou. Statistical analysis: Saliou. Administrative/technical/material support: Dirks, Krings. Study supervision: Dirks, Krings.

\section{Correspondence}

Guillaume Saliou, University Hospital, 78 Rue du Général Leclerc, le Kremlin Bicêtre 94275, France. email: guillaume. saliou@bct.aphp.fr. 